\title{
Permanência no mercado de trabalho e satisfação com a vida na velhice
}

\author{
Permanence in the labour market and life satisfaction in old age
}

Pricila Cristina Correa Ribeiro ${ }^{1}$

Daniele Soares Queiroz Almada ${ }^{1}$

Jéssica Faria Souto ${ }^{1}$

Roberto Alves Lourenço ${ }^{2}$

${ }^{1}$ Departamento de Psicologia, Faculdade de Filosofia e Ciência Humanas, Universidade Federal de Minas Gerais. Av. Antônio Carlos 667, Pampulha. 31270-901 Belo Horizonte MG Brasil. pricilaribeiro@ufmg.br

${ }^{2}$ Faculdade de Ciências Médicas, Universidade do Estado do Rio de Janeiro. Rio de Janeiro RJ Brasil.
Abstract Population aging is a global trend that has drawn attention to policies to encourage a productive life and delayed retirement. Thus, it is necessary to expand our understanding of the effects of work on health indicators and well-being in old age. The purpose of this study was to determine the association of permanency in the labor market with sociodemographic and medical factors and life satisfaction in elders. We used the database from Study FIBRA-RJ including elderly (aged $>65$ ) clients of a private health care plan who resided in northern districts of the municipality of Rio de Janeiro. Among the 626 participants, 82 (13,1\%) maintained paid jobs. Multiple logistic regression showed that the odds of remaining working among the elderly were higher for men; and those with 9 years of studies or more, and those with high income; and those with no disabling clinical conditions and with higher satisfaction with life. This study confirms that work activities in old age are associated with better social and physical health conditions. Moreover, we observed that the maintenance of work activities was associated with higher life satisfaction, independent of socioeconomic and clinical characteristics in old age.

Key words Aging, Elderly, Life Satisfaction, Employment
Resumo O envelhecimento da população é uma tendência mundial e chamou a atenção para as políticas de incentivo à vida produtiva e de adiamento da aposentadoria. Assim, cabe ampliar a compreensão dos efeitos do trabalho sobre os indicadores de saúde e bem-estar na velhice. $O$ objetivo deste estudo foi verificar a associação da permanência no mercado de trabalho com fatores sociodemográficos, clinicos e de satisfação com a vida em idosos. Utilizou-se a base de dados do Estudo FIBRA-RJ que inclui idosos (idade $\geq 65$ anos) clientes de uma operadora de saúde, residentes na zona norte do município do Rio de Janeiro. Dentre os 626 participantes, 82 (13,1\%) mantinham atividade de trabalho remunerado. Os resultados da regressão logística múltipla mostraram que as chances de permanecer trabalhando eram maiores para homens; para aqueles com 9 anos ou mais de estudos; com alta renda; sem condições clínicas crônicas incapacitantes e aqueles com maior satisfação com a vida. Este estudo confirma a associação das atividades de trabalho na velhice com melhores condições sociais e de saúde física. Além disso, observou-se que a manutenção das atividades de trabalho estava associada com a maior satisfação com a vida independente das características socioeconômicas e clínicas na velhice. Palavras-chave Envelhecimento, Idosos, Satisfação com a vida, Emprego 


\section{Introdução}

O Brasil sofre um processo acelerado de envelhecimento populacional e vai deixando de ser um país com uma população predominantemente jovem ${ }^{1}$. A redução da população em idade ativa é uma das consequências da transição demográfica, e o seu impacto social é forte o suficiente para gerar projeções do início da contração desta força de trabalho para meados de $2020^{2}$. Paralelo a este fenômeno, ocorre o aumento da proporção de idosos no mercado de trabalho, levando pesquisadores a questionar, nas últimas décadas, se estamos preparados para absorver o contingente populacional que pretende manter-se ocupado até a última fase da vida ${ }^{3,4}$.

Segundo o Instituto Brasileiro de Geografia e Estatística $^{5}$, a taxa de idosos brasileiros inseridos no mercado de trabalho em 2013 era de 27,4\% e subiu para $29,1 \%$ em $2014^{6}$. O aumento de idosos no mercado de trabalho vem sendo observado no país, mesmo em momentos nos quais ocorre uma diminuição da empregabilidade para as demais faixas etárias.

O nível de ocupação de pessoas de 60 anos ou mais de idade é ainda maior entre aqueles que não dispõem de aposentadoria ou pensão; em 2013, por exemplo, esta taxa era de $45,1 \%{ }^{5}$. Portanto, a ocupação na velhice parece associada à necessidade de manutenção ou melhora do rendimento familiar nesta fase da vida. Nesse levantamento do IBGE, embora a principal fonte de rendimento da população idosa fosse a aposentadoria ou pensão $(67,6 \%)$, o trabalho contribuía com 28,3\% da composição do rendimento nesse grupo ${ }^{5}$. A necessidade de prover a família, associada à perda do poder aquisitivo ocasionado pelos baixos valores dos benefícios previdenciários, podem explicar a permanência de idosos no mercado de trabalho. Não obstante, estudos apontaram outros fatores que contribuem para a manutenção das atividades laborais na velhice, entre eles, às experiências de prazer associadas à ocupação; a utilização do tempo livre com a prática de atividades física e/ou mentalmente estimulantes; o reconhecimento social e a manutenção de vínculos sociais ${ }^{7-10}$.

Tradicionalmente, o termo aposentadoria remete à velhice, mesmo que se considere que indivíduos aposentados também são representados por pessoas não idosas ${ }^{11}$. Aposentadoria refere-se ainda ao recolhimento do indivíduo velho, na qual o sujeito retorna ao interior da habitação e não é mais considerado ativo na sociedade ${ }^{12}$. Em entrevista com seis professores universitários, entre 61 e 88 anos, a fim de compreender o que os mantêm na atividade docente, Moreira ${ }^{13}$ constatou que todos eles, por considerarem o trabalho como central à vida, sentem a velhice como perda e a aposentadoria, quando sinônimo de não trabalho, como expressão da incapacidade. Contudo, tem aumentado, consideravelmente, a desvinculação entre velhice e aposentadoria, fato compatível com as mudanças no imaginário social de que nem todos os aposentados estão em idade avançada e de que a velhice não é sinônimo de aposentadoria ${ }^{14}$.

De acordo com Schwingel et al. ${ }^{15}$, os idosos que permanecem ocupados e ativos após a aposentadoria e/ou realizam trabalhos voluntários apresentaram melhor desempenho cognitivo, maior satisfação e bem-estar com a vida, e permanecem independentes em suas atividades diárias. Similarmente, outros estudos mostraram que o engajamento em trabalhos voluntários na velhice estava associado a ganhos, como melhor satisfação com a vida, autoestima positiva, melhor saúde física, autoavaliação positiva da saúde, melhora da depressão e redução do risco de mortalidade $\mathrm{t}^{7,16-18}$.

Tais artigos ressaltam a importância do trabalho após a aposentadoria, estabelecendo que, além de ser responsável pelo bom condicionamento físico e mental, proporciona ainda ao sujeito um senso de utilidade e bem-estar. Contudo, a relação entre a manutenção de trabalho remunerado e a satisfação com a vida na velhice não é consensual na literatura. Em estudo de Okamoto $^{19}$, a associação entre satisfação com vida e o trabalho remunerado não foi encontrada para os homens idosos investigados, esta associação foi obtida somente para as mulheres. No estudo de Nakahara ${ }^{20}$, esta associação não foi significativa para os idosos que realizavam trabalhos remunerados, somente para os que mantinham trabalhos não remunerados. Este autor argumentou que a permanência dos idosos em atividades remuneradas seria resultante de piores condições socioeconômicas e, portanto, não influenciaria positivamente no bem-estar na velhice. Nesta direção, cabe ampliar o estudo dos determinantes da permanência de uma vida ativa em idades mais avançadas, principalmente em países como o Brasil, onde fatores socioeconômicos impactam de forma significativa as escolhas na velhice. Além disso, cabe investigar se ocupações remuneradas entre idosos estão associadas à satisfação com a vida independente das condições socioeconômicas. O presente estudo investigou a associação da permanência de idosos no mercado de 
trabalho com fatores socioeconômicos e clínicos e de satisfação com a vida na velhice.

\section{Métodos}

Para compor a população de estudo foi utilizada a base de dados do estudo Fragilidade em Idosos Brasileiros - Seção Rio de Janeiro (FIBRA-RJ), que avaliou uma amostra de clientes idosos de uma operadora privada de saúde. Os detalhes da metodologia do estudo foram publicados em outro artigo ${ }^{21}$. Resumidamente, os critérios de inclusão foram ser cliente, há pelo menos 12 meses, da operadora de saúde; ter 65 anos ou mais de idade; e ser morador em um dos bairros da Zona Norte da cidade do Rio de Janeiro. De um total de 9.769 indivíduos que preencheram esses critérios selecionou-se uma amostra estratificada por sexo e faixa etária. Utilizou-se como estratégia uma amostragem aleatória inversa para uma previsão de preenchimento de 900 questionários. Para os estratos de homens e mulheres de 95 ou mais de idade realizou-se um censo.

Os 847 idosos que responderam ao protocolo do estudo FIBRA-RJ foram convidados a responder, por telefone, ao inventário de ocupação aplicado no ano seguinte à coleta de dados da linha de base. Destes, foram excluídos 81 idosos que apresentavam prejuízo cognitivo e/ou funcional graves e cuja participação no estudo FIBRA-RJ se deu a partir do relato de um informante substituto. Além disso, 78 participantes recusaram-se a responder ao questionário de ocupação e outros 62 foram considerados perdas devido a óbito ou por não serem mais localizados após a entrevista presencial. Portanto, no presente estudo, foram analisados os registros de 626 participantes do estudo FIBRA-RJ. Cabe acrescentar que dentre estes últimos, 12 idosos não responderam às perguntas sobre satisfação com a vida de forma que seus dados foram incluídos nas análises da associação de permanência no mercado de trabalho com as condições clínicas e sociodemográficas, mas não nas análises brutas e ajustadas para verificar associação de trabalho com a satisfação com a vida.

As características sociodemográficas incluíram as seguintes informações: idade; sexo; total de anos de estudo; e renda pessoal. Foram obtidos autorrelatos da presença de doenças crônicas (acidente vascular cerebral [AVC], câncer, artrite, doença pulmonar obstrutiva crônica [DPOC], depressão, osteoporose e coronariopatia) diagnosticadas por um médico no último ano.
Foi utilizado um questionário para avaliar a intensidade de satisfação com a vida referenciada a domínios ${ }^{22}$. Os domínios avaliados foram: satisfação com a vida atual; satisfação com a vida atual, comparado com outras pessoas da mesma idade; satisfação com a memória; satisfação com a capacidade para fazer coisas de todo dia; satisfação com as amizades e relações familiares; satisfação com o ambiente que vive; satisfação com o acesso aos serviços de saúde; satisfação com os meios de transporte. Para cada domínio o respondente deveria classificar seu nível de satisfação escolhendo entre uma das seguintes opções de resposta: pouco; mais ou menos; muito.

As informações sobre a ocupação foram obtidas por meio de questionário com perguntas semiestruturadas sobre as atividades de trabalho realizadas ao longo da vida. A variável dependente deste estudo intitulada "permanência no mercado de trabalho" foi obtida com a resposta à pergunta: "o senhor(a) exerce alguma atividade de trabalho?”. Além desta pergunta, outras questões sobre ocupação permitiram identificar se a atividade de trabalho exercida pelo respondente se referia a uma ocupação remunerada. Estas perguntas foram: 1) "Qual é a sua principal ocupação?" 2) "Esta atividade é a mesma ocupação exercida ao logo da vida?" e 3 ) "Descreva a(s) atividade(s) que você desenvolve no seu dia a dia de trabalho". A caracterização da ocupação principal foi feita a partir de uma lista pré-definida composta pelas categorias da Classificação Brasileira de Ocupação $(\mathrm{CBO})^{23}$, proposta pelo Ministério do Trabalho e Emprego. Segundo esta classificação a ocupação seria um conceito elaborado para englobar as atividades exercidas pelos indivíduos em seu dia-a-dia de trabalho, que pode ou não envolver um vínculo empregatício.

Para verificar a associação entre a permanência no mercado de trabalho e as características sociodemográficas, clínicas e de satisfação foram realizadas análises de associação com teste $\chi^{2}$. Para estimar as razões de chance (e seus respectivos intervalos de confiança) para associação da permanência no mercado de trabalho com características sociodemográficas, presença de patologias crônicas e satisfação com a vida foi realizada regressão logística múltipla.

Para análise dos dados foram utilizados o programa Statistical Package for the Social Sciences (SPSS) versão 18.0. Todos os resultados apresentados de frequências relativas e de medidas de associação foram ponderados pelo peso amostral.

O estudo foi autorizado pelo Comitê de Ética em Pesquisa do Hospital Universitário Pedro 
Ernesto e pela Comissão de Ética em Pesquisa da Sub-Reitoria de Pós-Graduação e Pesquisa da Universidade do Estado Rio de Janeiro.

\section{Resultados}

Dos 626 participantes investigados no presente estudo, $82(13,1 \%)$ exerciam atividade de trabalho, enquanto $544(86,9 \%)$ pararam de trabalhar. A frequência da permanência no mercado de trabalho é mostrada na Tabela 1, segundo as características sociodemográficas e clínicas. Entre os que ainda trabalhavam, observou-se o predomínio de homens (55\%), de 65 a 74 anos de idade $(58,4 \%)$, com treze anos ou mais de estudo (45,9\%) e renda superior a 5,1 salários $(66,3 \%)$. No grupo de idosos que pararam de trabalhar constatou-se um predomínio de mulheres (69,9\%), de 75 a 79 anos de idade $(25,3 \%)$, com nove a doze anos de estudo $(34,3 \%)$ e renda também superior a 5,1 salários (49,3\%).

Houve menor prevalência de todas as patologias estudadas no grupo que ainda trabalhava comparado ao que parou de trabalhar (Tabela 1). Hipertensão arterial sistêmica e diabetes mellitus foram exceções, sem atingir significância estatística. Uma melhor condição de saúde está positivamente associada com o trabalho.

O grupo de idosos que ainda trabalhava relatou estar mais satisfeito com a vida, comparado com aqueles que pararam de trabalhar, exceto para o domínio de satisfação com os meios de transporte. A associação da permanência no mercado de trabalho com satisfação referenciada a domínios é mostrada na Tabela 2.

A Tabela 3 descreve os resultados da regressão logística múltipla e mostra que as chances de permanecer trabalhando eram maiores para homens comparados às mulheres; para aqueles com 9 anos ou mais de estudos comparado aqueles com baixa escolaridade (não estudou ou tinha até 4 anos de estudos); e para aqueles com alta renda comparado aos que dispunham de renda pessoal de até dois salários mínimos, independente das condições clínicas e da satisfação com a vida.

$\mathrm{O}$ aumento da idade e a presença de doenças cardíacas, AVC, DPOC, depressão e osteoporose estiveram associados a menores chances de permanência nas atividades de trabalho. A associação com a presença de câncer $(\mathrm{p}=0,643)$ não foi mantida após ajuste pelas demais patologias, características sociodemográficas e a satisfação com a vida.
Tabela 1. Permanência no mercado de trabalho segundo características socioeconômicas e clínicas (N $=626)$.

\begin{tabular}{|c|c|c|}
\hline \multirow{3}{*}{ Variável } & \multicolumn{2}{|c|}{$\begin{array}{c}\text { Permanência no } \\
\text { mercado de trabalho }\end{array}$} \\
\hline & Sim & Não \\
\hline & $\mathbf{N}(\%)$ & $\mathbf{N}(\%)$ \\
\hline \multicolumn{3}{|l|}{$\mathrm{Sexo}^{*}$} \\
\hline Feminino & $41(45,0)$ & $396(69,9)$ \\
\hline Masculino & $41(55,0)$ & $148(30,1)$ \\
\hline \multicolumn{3}{|l|}{ Faixas etárias ${ }^{*}$} \\
\hline $65-69$ & $24(28,7)$ & $90(15,6)$ \\
\hline $75-79$ & $15(19,5)$ & $132(25,3)$ \\
\hline $70-74$ & $26(29,7)$ & $136(23,7)$ \\
\hline $80-84$ & $12(15,7)$ & $111(21,8)$ \\
\hline$\geq 85$ & $5(6,4)$ & $75(13,7)$ \\
\hline \multicolumn{3}{|l|}{ Escolaridade $^{*}$} \\
\hline $0-4$ & $11(13,7)$ & $101(18,3)$ \\
\hline $5-8$ & $13(14,9)$ & $111(20,4)$ \\
\hline $9-12$ & $21(25,5)$ & $187(34,3)$ \\
\hline$\geq 13$ & $37(45,9)$ & $145(27,0)$ \\
\hline \multicolumn{3}{|l|}{ Renda ${ }^{\text {*\#蝶 }}$} \\
\hline $0-2$ & $8(9,7)$ & $77(14,2)$ \\
\hline $2,1-5$ & $19(24,0)$ & $192(36,4)$ \\
\hline$>5,1$ & $51(66,3)$ & $255(49,3)$ \\
\hline \multicolumn{3}{|l|}{ Coronariopatia $^{*}$} \\
\hline Não & $77(93,8)$ & $468(85,6)$ \\
\hline $\operatorname{Sim}$ & $5(6,2)$ & $76(14,4)$ \\
\hline \multicolumn{3}{|l|}{$\mathrm{AVC}^{*}$} \\
\hline Não & $80(97,4)$ & $517(95,0)$ \\
\hline $\operatorname{Sim}$ & $2(2,6)$ & $27(5,0)$ \\
\hline \multicolumn{3}{|c|}{ Hipertensão Arterial $^{* * *}$} \\
\hline Não & $28(33,9)$ & $179(33,0)$ \\
\hline $\operatorname{Sim}$ & $54(66,1)$ & $365(67,0)$ \\
\hline \multicolumn{3}{|l|}{ Diabetes mellitus ${ }^{* * *}$} \\
\hline Não & $63(76,4)$ & $423(78,1)$ \\
\hline Sim & $19(23,6)$ & $121(21,9)$ \\
\hline \multicolumn{3}{|l|}{ Cancer $^{* *}$} \\
\hline Não & $77(93,8)$ & $519(95,3)$ \\
\hline $\operatorname{Sim}$ & $5(6,2)$ & $25(4,7)$ \\
\hline \multicolumn{3}{|l|}{ Artrite ou Artrose ${ }^{*}$} \\
\hline Não & $63(77,3)$ & $337(62,6)$ \\
\hline Sim & $19(22,7)$ & $207(37,4)$ \\
\hline \multicolumn{3}{|l|}{ DPOC $^{*}$} \\
\hline Não & $80(97,3)$ & $508(93,5)$ \\
\hline Sim & $2(2,7)$ & $36(6,5)$ \\
\hline \multicolumn{3}{|l|}{ Depressão* } \\
\hline Não & $77(94,0)$ & $462(85,2)$ \\
\hline Sim & $5(6,0)$ & $82(14,8)$ \\
\hline \multicolumn{3}{|l|}{ Osteoporose } \\
\hline Não & $70(86,6)$ & $388(72,0)$ \\
\hline Sim & $12(13,4)$ & $156(28,0)$ \\
\hline $\begin{array}{l}\mathrm{N}(\%) \text { : número de indiv } \\
\text { (frequência relativa pon } \\
<0,001 \text {; }{ }^{* *} \text { p-valor }<0 \text {, } \\
\text { salário mínimo: } \mathrm{R} \$ 465 \\
\text { realização do estudo. }\end{array}$ & $\begin{array}{l}\text { ostra não } p \\
\text { peso amost } \\
\text { lor }>0,1 ; \#: \\
\text { le referência }\end{array}$ & $\begin{array}{l}\text { onderada } \\
\text { cal); }{ }^{\star} p \text {-valor } \\
N=602 ; \ddagger: \\
\text { no período de }\end{array}$ \\
\hline
\end{tabular}


Tabela 2. Satisfação com a vida referenciada a domínios segundo a situação no mercado de trabalho $(\mathrm{N}=614)$.

\begin{tabular}{|c|c|c|c|c|}
\hline \multirow{4}{*}{ Domínios de Satisfação } & \multicolumn{4}{|c|}{ Permanência no mercado de trabalho } \\
\hline & \multirow{3}{*}{$\begin{array}{c}\text { Sim } \\
\text { N (\%) } \\
\text { Pouco/mais } \\
\text { ou menos } \\
\text { N (\%) }\end{array}$} & \multirow{2}{*}{\multicolumn{3}{|c|}{$\begin{array}{c}\text { Não } \\
\end{array}$}} \\
\hline & & & & \\
\hline & & $\begin{array}{l}\text { Muito } \\
\text { N (\%) }\end{array}$ & $\begin{array}{c}\text { Pouco/mais } \\
\text { ou menos } \\
\text { N (\%) }\end{array}$ & $\begin{array}{l}\text { Muito } \\
\text { N (\%) }\end{array}$ \\
\hline Vida atual $^{* *}$ & $23(28)$ & $59(72,1)$ & $262(49,2)$ & $270(50,8)$ \\
\hline $\begin{array}{l}\text { Vida atual, comparado com outras pessoas } \\
\text { da mesma idade }\end{array}$ & $13(15,9)$ & $69(84,2)$ & $148(27,9)$ & $383(72,3)$ \\
\hline Memória* & $39(47,6)$ & $43(51,2)$ & $277(52,1)$ & $255(48,3)$ \\
\hline Capacidade para fazer coisas de todo dia* & $16(19,5)$ & $66(79,7)$ & $197(37)$ & $335(62,8)$ \\
\hline Amizades e relações familiares* & $20(24,4)$ & $62(75,2)$ & $167(31,4)$ & $365(68,6)$ \\
\hline Ambiente onde vive ${ }^{\star}$ & $32(39)$ & $50(61)$ & $255(47,9)$ & $277(52,2)$ \\
\hline Serviços de saúde ${ }^{*}$ & $32(39)$ & $50(60,5)$ & $263(49,4)$ & $269(51,2)$ \\
\hline Meios de transporte ${ }^{\star *}$ & $39(47,6)$ & $43(52,7)$ & $256(48,1)$ & $276(51,9)$ \\
\hline
\end{tabular}

${ }^{*}$-valor $<0,001 ;{ }^{* *}$ p-valor $>0,05$.

Quanto à satisfação com a vida, o modelo logístico indicou que idosos que permanecem no mercado de trabalho estão mais satisfeitos com a vida que aqueles que pararam de trabalhar, independente das características sociodemográficas e das condições clínicas.

\section{Discussão}

No presente estudo, a permanência no mercado de trabalho após os 65 anos esteve associada ao sexo masculino, às faixas etárias mais jovens, à maior escolaridade e à maior renda. Além disso, esta manutenção do trabalho remunerado entre os idosos mostrou-se associada a melhores condições de saúde física e maior satisfação com a vida na velhice.

No que diz respeito aos fatores sexo e idade, Wajnman et al..$^{24}$ destacam que homens e mulheres têm, ao longo do ciclo da vida, comportamentos e papéis muito distintos ante o mercado de trabalho, chegando, assim, à velhice com níveis de atividade muito diferenciados. De acordo com Rocha-Coutinho ${ }^{25}$, estudos realizados em diferentes países revelaram que tanto os homens quanto as mulheres pensavam que a casa e os filhos eram responsabilidades femininas, cabendo aos homens o sustento financeiro da família. Esta é uma realidade cultural ainda preservada, principalmente pelas coortes mais velhas nas quais o papel de cuidador é, predominantemente, ocu- pado pelas mulheres. A exemplo disso, sabe-se que o trabalho doméstico e o cuidado da prole continuam sendo atribuídos à mulher, prioritariamente ${ }^{26,27}$. Contudo, mudanças na chefia e sustento das famílias sofreram significativas alterações em função dos novos papeis femininos no mercado de trabalho e na estrutura familiar composta por casais sem filhos ${ }^{28,29}$. No Brasil, a realidade se modificou também em decorrência da legislação, cujas garantias previdenciárias e assistenciais aos idosos beneficiaram muitas mulheres que, embora não tenham contribuído formalmente para previdência social, tiveram um rendimento garantido na velhice ${ }^{30-32}$.

Quanto à influência da idade, verificou-se que a taxa de idosos que permaneciam ativos no mercado de trabalho diminuiu com a idade, passando de $58,4 \%$ na faixa de $65-74$ anos para 19,5\% entre 75 e 79 anos de idade. No Brasil, estes resultados já vinham sendo observados nas últimas décadas. Por exemplo, Camarano et al. ${ }^{33}$ observaram uma redução na taxa de permanência no mercado de trabalho, que era da ordem de $47 \%$, aos 65 anos de idade, para $22,1 \%$ aos 75 anos. Da mesma forma, Wajnman et al. ${ }^{24}$ também apontaram que as taxas de atividade declinaram fortemente com a idade. Estes achados são compatíveis com dados recentes do $\mathrm{IBGE}^{6}$ que mostraram taxas maiores de ocupação para homens, comparado às mulheres, e decrescentes à medida que aumenta a faixa etária para ambos os sexos. Essa taxa foi de $41,9 \%$ para os homens 
Tabela 3. Razões de chances com intervalo de $95 \%$ de confiança para associação da permanência no mercado de trabalho com características sociodemográficas, presença de patologias crônicas e satisfação com a vida $(\mathrm{N}=614)$.

\begin{tabular}{|c|c|c|}
\hline Variáveis & OR (IC95\%) & p-valor \\
\hline \multicolumn{3}{|l|}{ Sexo } \\
\hline Masculino & 1,00 & \\
\hline Feminino & $0,387(0,328-0,456)$ & 0,000 \\
\hline \multicolumn{3}{|l|}{ Faixa etária } \\
\hline $75-79$ & 1,00 & \\
\hline $80-84$ & $0,449(0,334-0,604)$ & 0,000 \\
\hline$\geq 85$ & $0,716(0,514-0,997)$ & 0,048 \\
\hline \multicolumn{3}{|l|}{ Escolaridade } \\
\hline $0-4$ & 1,00 & \\
\hline $5-8$ & $0,932(0,711-1,222)$ & 0,612 \\
\hline $9-12$ & $1,580(1,235-2,021)$ & 0,000 \\
\hline$\geq 13$ & $1,891(1,551-2,305)$ & 0,000 \\
\hline \multicolumn{3}{|l|}{$\operatorname{Renda}^{*}$} \\
\hline $0-2$ & 1,00 & \\
\hline $2,1-5$ & $1,407(1,059-1,868)$ & 0,018 \\
\hline$>5,1$ & $1,839(1,511-2,238)$ & 0,000 \\
\hline \multicolumn{3}{|l|}{ Satisfação com a vida } \\
\hline Muito & 1,00 & \\
\hline Pouco/Mais ou Menos & $0,527(0,446-0,624)$ & 0,000 \\
\hline \multicolumn{3}{|l|}{ Coronariopatia } \\
\hline Não & 1,00 & \\
\hline Sim & $0,360(0,268-0,483)$ & 0,000 \\
\hline \multicolumn{3}{|l|}{ AVC } \\
\hline Não & 1,00 & \\
\hline Sim & $0,556(0,353-0,877)$ & 0,012 \\
\hline \multicolumn{3}{|l|}{ Câncer } \\
\hline Não & 1,00 & \\
\hline Sim & $1,096(0,795-1,511)$ & 0,577 \\
\hline \multicolumn{3}{|l|}{ Artrite ou Artrose } \\
\hline Não & 1,00 & \\
\hline Sim & $0,732(0,607-0,883)$ & 0,001 \\
\hline \multicolumn{3}{|l|}{ DPOC } \\
\hline Não & 1,00 & \\
\hline Sim & $0,565(0,367-872)$ & 0,010 \\
\hline \multicolumn{3}{|l|}{ Depressão } \\
\hline Não & 1,00 & \\
\hline Sim & $0,454(0,329-0,629)$ & 0,000 \\
\hline \multicolumn{3}{|l|}{ Osteoporose } \\
\hline Não & 1,00 & \\
\hline Sim & $0,705(0,561-0,886)$ & 0,003 \\
\hline $\begin{array}{l}\text { OR (IC95\%): razão de chance } \\
\text { incluindo as variáveis demogr } \\
\text { vida atual, ponderado pelo pe } \\
\text { de } 95 \%) ;{ }^{*} 24 \text { idosos não forn } \\
\text { e para estes casos foi imputad } \\
\text { população de estudo. }\end{array}$ & $\begin{array}{l}\text { s do modelo de regressão } \\
\text { áficas, clínicas e de satisfac } \\
\text { so amostral (intervalo de } \\
\text { eceram informação sobre } \\
\text { a a média da renda relatad }\end{array}$ & $\begin{array}{l}\text { logística } \\
\text { cão com a } \\
\text { confiança } \\
\text { a renda } \\
\text { a pela }\end{array}$ \\
\hline
\end{tabular}

e de $18,9 \%$ para as mulheres com idade igual ou superior a 60 anos, mas caiu para 30,0\% para os homens com 65 anos ou mais e $23,5 \%$ para aqueles com 70 anos ou mais. Cabe destacar que mesmo decrescendo com o aumento da idade, o nível de ocupação dos homens é superior ao das mulheres em todas as faixas etárias. Um fator que pode influenciar na prevalência dos homens idosos que retornam ao trabalho, após a aposentadoria, é a crescente quantidade de famílias dependentes da renda do idoso que, no papel de provedor principal, acaba optando por adiar sua aposentadoria ou voltam à atividade laboral para reforçar o orçamento doméstico ${ }^{34-36}$.

Nas classes sociais mais altas, a aposentadoria é definida pela queda da renda, uma vez que remete à perda das gratificações ${ }^{10,37}$. Assim, tornam-se grandes as chances de permanecerem ativos aqueles idosos mais bem qualificados. A presente investigação encontrou que $45,9 \%$ dos idosos que continuaram trabalhando após a aposentadoria possuíam treze anos ou mais de escolaridade; este percentual foi de $27 \%$ no grupo que saiu do mercado de trabalho. Pressupõe-se que os idosos com maior escolaridade, provavelmente, tiveram acesso a ocupações mais qualificadas e optaram pela permanência no mercado de trabalho, motivados tanto pelo rendimento, quanto pela satisfação com as atividades laborais e suas conquistas.

As reformas nos sistemas previdenciários $\mathrm{e}$ as políticas de admissão de grupos específicos de trabalhadores, tanto em países desenvolvidos quanto nos de renda média como o Brasil, atuam como incentivos ao adiamento da aposentadoria e à permanência da população idosa no mercado de trabalho formal ${ }^{38}$. No entanto, os trabalhadores mais velhos, incluindo os aposentados que legalmente não podem receber benefícios como seguro desemprego, dispõem de condições desfavoráveis de empregabilidade, como remunerações mais baixas e/ou maiores chances de demissão, quando comparados aos mais joven ${ }^{39}$. Em virtude dessa valorização dos funcionários mais jovens e com maior capacidade de produção no mercado formal, o trabalho informal é visto como boa oportunidade para aqueles que querem permanecer trabalhando e complementando a renda após a aposentadoria ${ }^{11,29}$. Assim, os idosos têm sido atraídos para a realização do trabalho informal, de tempo parcial, temporário e autônomo.

Com relação aos indicadores de saúde, verificou-se que as condições clínicas que aumentam as chances de perdas funcionais, como a coro- 
nariopatia, AVC, artrite/artrose, DPOC, depressão, osteoporose estavam associadas à saída dos idosos do mercado de trabalho após a aposentadoria. Já a hipertensão e o diabetes não foram relacionadas à permanência dos idosos no mercado de trabalho. Em estudo de Boot et al..$^{40}$ sobre os preditores de saída de idosos do mercado de trabalho, os autores também apontaram os mais velhos e com doença crônica, além daqueles com poucos recursos psicossociais no trabalho, como os mais prováveis de parar de trabalhar. A partir disso, pode-se concluir sobre a importância da manutenção da saúde física para a permanência no mercado de trabalho na velhice e para o retorno a estas atividades após a aposentadoria.

Quanto à satisfação com a vida, constatou-se que idosos que continuaram exercendo atividade de trabalho remunerado na velhice relataram estar mais satisfeitos com a vida, em suas diversas dimensões (com a vida em geral, com as relações sociais, aspectos cognitivos e funcionais e outros) comparado àqueles que pararam de trabalhar. Em estudo realizado por Nakahara ${ }^{20}$, com idosos japoneses, não foi encontrada associação do trabalho remunerado com a satisfação com a vida, sendo o baixo nível socioeconômico utilizado para explicar a ausência de associação entre estas variáveis. Ao contrário, na população do presente estudo o trabalho remunerado na velhice foi associado com melhores condições socioeconômicas. Assim, supõe-se que, enquanto entre idosos de baixa renda a necessidade de trabalhar parece não permitir uma maior satisfação com a vida, nas classes com maior poder aquisitivo, a manutenção do trabalho pode significar a manutenção do papel social e da qualidade de vida e, consequentemente, maior satisfação com a vida na velhice. Nesta direção, outros estudos ${ }^{41-43}$ mostraram que a manutenção da ocupação remunerada estava associada a desfechos psicossociais positivos na velhice, como as interações sociais e o bem-estar psicológico. São necessários mais estudos com idosos das classes socioeconômicas mais baixas vivendo em países de média e baixa renda para compreender se a permanência no mercado de trabalho, motivada, principalmente, pelas dificuldades financeiras, impacta a satisfação com a vida na velhice.

O benefício do trabalho voluntário, seja para indicadores objetivos da saúde ${ }^{16,17}$, seja para aspectos subjetivos, como a satisfação com a vida e a autoavaliação positiva da saúde geral ${ }^{18}$, foi amplamente demonstrado na literatura internacional. O presente estudo não analisou a prática de trabalhos voluntários, sendo o trabalho remu- nerado escolhido como objeto de investigação porque este tipo de ocupação é tido na literatura gerontológica como uma categoria de atividade que se diferencia de outras produtivas, como as realizadas apenas no próprio ambiente doméstico ou com o propósito de ajudar os outros, como ocorre com o cuidado informal e o voluntaria$\mathrm{do}^{44}$. Assim, embora trabalhos voluntários e remunerados estejam ambos dentro de uma esfera de ocupação social para os idosos, supõe-se que são ocupações de natureza distinta e sugere-se que novas investigações de base populacional comparem seus benefícios entre os idosos brasileiros.

Além disso, a direcionalidade entre as variáveis de trabalho e a satisfação com a vida não foi o foco da presente investigação que seguiu delineamento transversal. Estudos longitudinais deverão ser conduzidos para identificar se a manutenção do trabalho na velhice leva a maior satisfação com a vida ou se seria a condição de avaliação mais positiva que funcionaria como um indicador do perfil do idoso que se mantem engajado com atividades de trabalho. Cabe considerar ainda que o perfil socioeconômico dos idosos entrevistados, com maior escolaridade e maior renda que da população idosa brasileira em geral, limita a generalização das associações obtidas entre as características sociodemográficas e clínicas e a permanência no mercado de trabalho. O perfil da população de estudo está associado à população fonte do estudo, constituída por clientes de uma operadora de saúde. Esta população pode representar os idosos brasileiros com acesso ao setor privado de saúde que vem sendo descrito como uma população com melhores condições de escolaridade e de renda e, consequentemente, maior acesso aos cuidados em saúde que a população idosa com cobertura apenas do sistema público de saúde ${ }^{45}$.

No campo gerontológico, o estudo entre satisfação e trabalho remunerado em idosos prioriza a compreensão da satisfação dos idosos com o próprio trabalho visando identificar os determinantes da saída precoce do mercado de traba$\mathrm{lho}^{42}$. Os achados do presente estudo corroboram a literatura científica ${ }^{31,46-49}$ que aponta os fatores sociais como determinantes para escolha da continuidade das atividades laborais após aposentadoria. Além desta associação com condições sociais favoráveis, como melhor escolaridade e renda, o estudo ampliou a discussão sobre a associação da realização de ocupação remunerada na velhice com a maior satisfação com a vida. Assim, entende-se que o trabalho pode atuar, não 
só como garantia da sobrevivência do idoso e de seus familiares, mas também como fonte de satisfação, como elemento gerador de qualidade de vida. Estas atividades podem funcionar como estratégias associadas à manutenção do bem-estar e de uma atitude ativa dos indivíduos mais velhos. Nesse sentido, os resultados apresentados apontam para a necessidade da sociedade se organizar para ampliar as oportunidades de trabalho para a população que pretende se manter produtiva até a idade avançada.

\section{Colaboradores}

PCC Ribeiro trabalhou na concepção, delineamento e supervisão de coleta de dados do estudo; na análise e interpretação dos dados e na redação do artigo. DSQ Almada e JF Souto trabalharam na análise e interpretação dos dados e na redação do artigo. RA Lourenço trabalhou na concepção e delineamento do estudo e na revisão crítica da redação final do artigo. 


\section{Referências}

1. Instituto Brasileiro de Geografia e Estatística (IBGE). Síntese de indicadores sociais. Uma análise das condições de vida da população Brasileira. Estudos e pesquisas: Informação Demográfica e Socioeconômica. Rio de Janeiro: IBGE; 2009. no 26. [acessado 2016 Jun 21]. Disponível em: http://biblioteca.ibge.gov.br/visualizacao/livros/liv 42820.pdf

2. Nonato FJ, Pereira R, Nascimento PA, Araújo TC. O perfil da força de trabalho brasileira: trajetórias e perspectivas. In: Nonato FJ, organizador. Mercado de Trabatho, n. 51. Brasília: IPEA; 2012. p. 30-41.

3. Queiroz V, Ramalho H. A escolha ocupacional dos idosos no mercado de trabalho: evidências para o Brasil. Economia 2009; 10(4):817-848.

4. Pavani RMG. A imagem da velhice. In: Trevisan L, Castro, MCA, organizadores. Transformações no trabalho. São Paulo: Olho d'Água; 2002.

5. Instituto Brasileiro de Geografia e Estatística (IBGE). Síntese dos indicadores sociais: Uma análise das condições de vida da população brasileira: 2014. Estudos e pesquisas: Informação Demográfica e Socioeconômica. $\mathrm{n}$. 34. Rio de Janeiro: IBGE; 2014. [acessado $2016 \mathrm{Fev} 02$ ]. Disponível em: http://biblioteca.ibge.gov.br/visualizacao/livros/liv91983.pdf

6. Instituto Brasileiro de Geografia e Estatística (IBGE). Sintese de indicadores sociais: uma análise das condições de vida da população brasileira. Estudos e pesquisas: Informação Demográfica e Socioeconômica. Rio de Janeiro: IBGE; 2015. n. 35. [acessado 2016 Fev 02]. http:// biblioteca.ibge.gov.br/visualizacao/livros/liv95011.pdf

7. Musick MA, Wilson J. Volunteering and depression: the role of psychological and social resources in different age groups. Soc Sci Med 2003; 56(2):259-269.

8. Goulart Júnior E, Mergulhão LR, Canêo LC, Najm MB, Lunardelli MCF. Considerações sobre a terceira idade e o mercado de trabalho: questionamentos e possibilidades. RBCEH 2009; 3(6):429-437.

9. Vanzella E, Lima Neto EA, Silva CC. A terceira idade e o mercado de trabalho. RBCS 2011; 14(4):97-100.

10. Cockell FF. Idosos aposentados no mercado de trabalho informal: trajetórias ocupacionais na construção civil. Psicol Soc 2014; 26(2):461-471.

11. Cintra TS, Ribeiro DF, Andrade AS. O cotidiano de aposentados que continuam trabalhando de maneira informal na indústria calçadista: percepções sobre a aposentadoria e o trabalho atual. Cad Psicol Soc Trab 2010; 13(2):277-287.

12. Khoury HTT, Ferreira AJC, Souza RA, Matos AP, Barbagelata-Góes S. Por que aposentados retornam ao trabalho? O papel dos fatores psicossociais. Kairós Gerontologia 2010; 13(1):147-165.

13. Moreira JO. Imaginários sobre aposentadoria, trabalho, velhice: estudo de caso com professores universitários. Psicol Estud 2011; 4(16):541-550.

14. Luborsky M, Leblanc I. Cross-cultural perspectives on the concept of retirement: An analytic redefinition. $J$ Cross Cult Gerontol 2003; 18(4):251-327.

15. Schwingel A, Niti MM, Tang C, Ng TP. Continued work employment and volunteerism and mental well being of older adults: Singapore longitudinal ageing studies. Age Ageing 2009; 38(5):531-537.
16. Wilson J. Volunteering. Annu Rev Sociol 2000; 26:215240.

17. Okun MA, Yeung EWH, Brown S. Volunteering by older adults and risk of mortality: A meta-analysis. Psychol Aging 2013; 28(2):564-577.

18. Wu AMS, Tang CSK, Yan ECW. Post-retirement voluntary work and psychological functioning among older Chinese in Hong Kong. J Cross Cult Gerontol 2005; 20(1):27-45

19. Okamoto H. Productive activities and well-being among community-dwelling elderly. Nihon Koshu Eisei Zasshi 2009; 56(10):713-723.

20. Nakahara J. Effects of social activities outside the home on life satisfaction among elderly people living alone. IJPS 2013; 5(1):112-120.

21. Lourenço RA, Sanchez MA, Moreira VG, Ribeiro PCC, Perez M, Campos GC, Blavatsky I, Teldeschi ALG. Fragilidade em Idosos Brasileiros - Fibra-RJ: metodologia de pesquisa dos estudos de fragilidade, distúrbios cognitivos e sarcopenia. Revista HUPE 2015; 14(4):13-23.

22. Neri AL. Bienestar subjetivo en la vida adulta y en la vejez: rumbo a una Psicologia positiva en América Latina. RLP 2002; 43(1-2):55-74.

23. Brasil. Ministério do trabalho e emprego (MTE). Classificação Brasileira de Ocupações: $C B O$. Brasília: MTE; 2010.

24. Wajnman S, Oliveira AM, Oliveira EL. Os idosos no mercado de trabalho: tendências e consequências. In: Camarano AA, organizador. Os novos idosos brasileiros: muito além dos 60? Rio de Janeiro: IPEA; 2004. p. 453479.

25. Rocha-Coutinho ML. O papel de homens e mulheres na família: Podemos falar em reestruturação? Psicol Clin 2003; 15(2):93-107.

26. Instituto Brasileiro de Geografia e Estatística (IBGE). Síntese de indicadores sociais. Estudos e pesquisas: Informação Demográfica e Socioeconômica. n. 19. Rio de Janeiro: 2006. [acessado 2016 Fev 03]. Disponível em: http://biblioteca.ibge.gov.br/visualizacao/livros/ liv32725.pdf

27. Madalozzo R, Martins SR, Shiratori L. Participação no mercado de trabalho e no trabalho doméstico: Homens e mulheres têm condições iguais? Rev. Est. Fem. 2008; 18(2):547-566.

28. Camarano AA, Pasinato MT. Envelhecimento, pobreza e proteção social na América Latina. Texto para discussão no 1292. Rio de Janeiro: Instituto de Pesquisa Econômica Aplicada (IPEA); 2007.

29. Silva LCM. Trabalho e Família na Percepção de Mulheres Provedoras. Gênero na Amazônia 2012; 1:61-83.

30. Brasil. Lei $\mathrm{n}^{\circ} 10.741$ de $1^{\circ}$ de Outubro de 2003. Dispõe sobre o Estatuto do Idoso e dá outras providências. Diário Oficial da União 2003; 3 out.

31. Rocha EG. Estatuto do idoso: um avanço legal. Revista da UFG [periódico na Internet] 2003 Dez [acessado 2016 Mar 20]; 2(5):[cerca de 4 p.]. Disponível em: http://www.proec.ufg.br/revista_ufg/idoso/estat_legal. html

32. Camarano AA, Kanso S, Fernandes D. Envelhecimento populacional, perda da capacidade laborativa e políticas públicas brasileiras entre 1992 e 2011. Rio de Janeiro: 
Instituto de Pesquisa Econômica Aplicada (IPEA); 2013.

33. Camarano A, Beltrão KI, Pascom ARP, Medeiros M, Carneiro IG, Goldani AM, Vasconcelos AMN, Chagas AMR, Osório RG. Como vai o Idoso Brasileiro? Rio de Janeiro: Instituto de Pesquisa Econômica Aplicada (IPEA); 1999. [Texto para Discussão 681].

34. Peixoto CE. Aposentadoria: retorno ao trabalho e solidariedade familiar. In: Peixoto CE, organizador. Família e envelhecimento. Rio de Janeiro: Fundação Getúlio Vargas; 2004. p. 57-84.

35. Instituto Brasileiro de Geografia e Estatística (IBGE). IBGE: população brasileira envelhece em ritmo acelerado: 2008. [acessado 2015 Out 20]. Disponível em: http://www.ibge.gov.br/home/presidencia/noticias/ noticia_visualiza.php2.id_noticia=1272\&id_pagina

36. Oliveira MB, Silva NM. Participação de aposentados nas transferências de recursos familiares. $R C H$ 2012; 1(12):135-144.

37. Franca LHFP, Vaughan G. Ganhos e perdas: atitudes dos executivos brasileiros e neozelandeses frente à aposentadoria. Psicol. Estud. 2008; 13(2):207-216.

38. Furtado A. A participação do idoso no mercado de trabalho brasileiro. Consultoria Legislativa da Câmara dos Deputados. Brasília; 2005. [acessado 2016 Fev 03]. Disponível em: http://www2.camara.gov.br/publicacoes/ estnottec/tema8/2004_13576.pdf

39. Standing G. O precariado: a nova classe perigosa. Belo Horizonte: Autêntica Editora; 2013.

40. Boot CR, Deeg DJ, Abma T, Rijs KJ, van der Pas S, van Tilburg TG, van der Beek AJ. Predictors of having paid work in older workers with and without chronic disease: a 3-year prospective cohort study. J Occup Rehabil 2014; 24(3):563-572.

41. Matz-Costa C, Besen E, Boone James J, Pitt-Catsouphes M. Differential impact of multiple levels of productive activity engagement on psychological well-being in middle and later life. Gerontologist 2014; 54(2):277-289.

42. Dave D, Rashad I, Spasojevic J. The effects of retirement on physical and mental health outcomes. Southern Economic Journal; 2008 75(2):497-523.
43. Hao Y. Productive activities and psychological well-being among older adults. J Gerontol B Psychol Sci Soc Sci 2008; 63(2):S64-S72.

44. Klumb PL, Baltes MM. Time use of old and very old Berliners: Productive and consumptive activities as functions of resources. J Gerontol B Psychol Sci Soc Sci 1999; 54(5):S271-S278.

45. Malta DC, Moura EC, Oliveira M, Santos FP. Health insurance users: self-reported morbidity and access to preventive tests according to a telephone survey, Brazil, 2008. Cad Saude Publica 2011; 27(1):57-66.

46. Olesen SC, Butterworth P, Rodgers B. Is poor mental health a risk factor for retirement? Findings from a longitudinal population survey. Soc Psychiatry Psychiatr Epidemiol 2012; 47(5):735-744.

47. Giatti L, Barreto SM. Saúde, trabalho e envelhecimento no Brasil. Cad. Saúde Pública 2003; 19(3):759-771.

48. Flippen C, Tienda M. Pathways to retirement: Patterns of labor force participation and labor market exit among the pre-retirement population by race, hispanic origin, and sex. J Gerontol 2000; 55(1):14-27.

49. Parnes HS, Sommers DG. Shunning retirement: Work experience of men in their seventies and early eighties. J Gerontol 1994; 49(3):117-124.

Artigo apresentado em 15/04/2016

Aprovado em 25/08/2016

Versão final apresentada em 27/08/2016 